\title{
On the Chiroptical Behavior of Conjugated Multichromophoric Compounds of a New Pseudoaromatic Class: Bicolchicides and Biisocolchicides
}

\author{
Tiziana Funaioli ${ }^{1 *}$, Marino Cavazza ${ }^{1}$, Maurizio Zandomeneghi ${ }^{1}$, Francesco Pietra ${ }^{2}$ \\ 1 Dipartimento di Chimica e Chimica Industriale, Università di Pisa, Pisa, Italy, 2 Accademia Lucchese di Scienze, Lettere e Arti, Lucca, Italy
}

\begin{abstract}
Background: It is well known that, stemming from the mutual interplay between chromophores, circular dichroism (CD) is a powerful technique to deal with structural problems for both the small organic molecule and the biopolymer. However, quantitative interpretations of the spectroscopic and structural terms that give rise to the exciton couplet are usually presented for ideal cases, or a few CD bands only are taken into account, overlooking the role of the solvent medium.

Methodology/Principal Findings: Circular dichroism and UV absorption spectra were carried out for colchicide (3) and isocolchicide (6), as well as their coupling products, 10,10'-bicolchicide (2) and 9,9'-biisocolchicide (5), in both hydrogen bonding and non hydrogen bonding solvents, as well as $\mathrm{MeCN} / \mathrm{H}_{2} \mathrm{O}$ mixtures. A dramatic control by the solvent emerged, as even tiny changes in the composition of solvent mixtures, at ca 1 water molar fraction, induced a dramatic modification of their CD bands. A mutarotation phenomenon - long known for isocolchicine (8) - was also observed for 5 , and can be attributed to the interconversion between atropisomers $\left(R_{\mathrm{a} a}, 7 S\right),\left(R_{\mathrm{a}}, 7^{\prime} S\right)-5 \mathrm{a}$ and $\left(R_{\mathrm{a}}, 7 S\right),\left(S_{\mathrm{a}}, 7^{\prime} S\right)-5 \mathrm{~b}$.

Conclusions/Significance: Our data show that with molecules built on two structurally identical moieties which embody both hydrophilic and hydrophobic groups, even tiny changes in the composition of solvent mixtures cause a dramatic modification of the CD bands. Their analysis arrives at a qualitative rationalization of the observed CD couplets from the coupling of high energy transitions, while attempts at a quantitative interpretation of these phenomena through timedependent density functional theory allowed to reproduce satisfactorily the CD spectrum in the $300-450 \mathrm{~nm}$ region only. Failure with higher energies probably reflects currently inadequate specific theoretical treatments of the solvent medium.
\end{abstract}

Citation: Funaioli T, Cavazza M, Zandomeneghi M, Pietra F (2010) On the Chiroptical Behavior of Conjugated Multichromophoric Compounds of a New Pseudoaromatic Class: Bicolchicides and Biisocolchicides. PLoS ONE 5(5): e10617. doi:10.1371/journal.pone.0010617

Editor: Matthew H. Todd, University of Sydney, Australia

Received June 29, 2009; Accepted April 14, 2010; Published May 12, 2010

Copyright: ( $) 2010$ Funaioli et al. This is an open-access article distributed under the terms of the Creative Commons Attribution License, which permits unrestricted use, distribution, and reproduction in any medium, provided the original author and source are credited.

Funding: This work was supported by the University of Pisa and by MIUR-PRIN 2006-2007 (TF). The funders had no role in study design, data collection and analysis, decision to publish, or preparation of the manuscript.

Competing Interests: The authors have declared that no competing interests exist.

*E-mail: tiziana@dcci.unipi.it

\section{Introduction}

Circular dichroism (CD) is a powerful technique to deal with structural problems for both the small organic molecule and the biopolymer. This stems from largely the mutual interplay between chromophoric units, which affects the molecular properties. Under favorable circumstances, when two or more equivalent chromophores - which absorb light strongly in the same spectral region are present in a molecular frame at suitable mutual distance and orientation, the CD spectral features (e.g. exciton couplets) may offer a clue as to the stereochemistry of the molecule [1], [2].

Models of such systems, built from either classical electrodynamics [3], [4] or quantum mechanics [5], can also allow a quantitative interpretation of the spectroscopic and structural terms that give rise to the exciton couplet [6]-[8]. However, except in the near-to-ideal cases, success is often limited to a few bands only. In any case, as expected for calculations with electronically excited states, any quantitative interpretation is faced with problems. This is especially true when the conformational behavior of the molecule depends on the nature of the solvent, like for any theoretical treatment of solvent effects.
Here we present a notable example of solvent-dependent circular dichroism involving multichromophoric systems. It concerns 10,10'-bicolchicide (2) and 9,9'-biisocolchicide (5), where two identical colchicinoid $(\mathbf{1})$ or isocolchicinoid $(\mathbf{4})$ chromophores of known absolute configuration $\left(R_{a}, 7 S\right)$, are linked through a formally single covalent bond (Figure 1), establishing, as it will become clear in the following, a new pseudoaromatic class. These relatively large molecules (molecular mass 736) embody both hydrophilic and hydrophobic groups, which make them easily soluble in solvents like chloroform, acetonitrile and trifluoroethanol, while being sparingly soluble in water. We illustrate here how the coupling process with $\mathbf{1}$ or $\mathbf{4}$, by increasing the molecular complexity, gives rise to spectroscopic properties that do not show up with the starting molecules.

\section{Results}

Synthesis of the bicolchicides and biisocolchicides

Compounds $\mathbf{2}$ and $\mathbf{5}$ could be obtained by homocoupling of chlorocolchicides by using Semmelhack's recipe, i.e., by inducing the coupling reaction with stoichiometric amounts of $\mathrm{Ni}(\mathrm{COD})_{2}$ in 


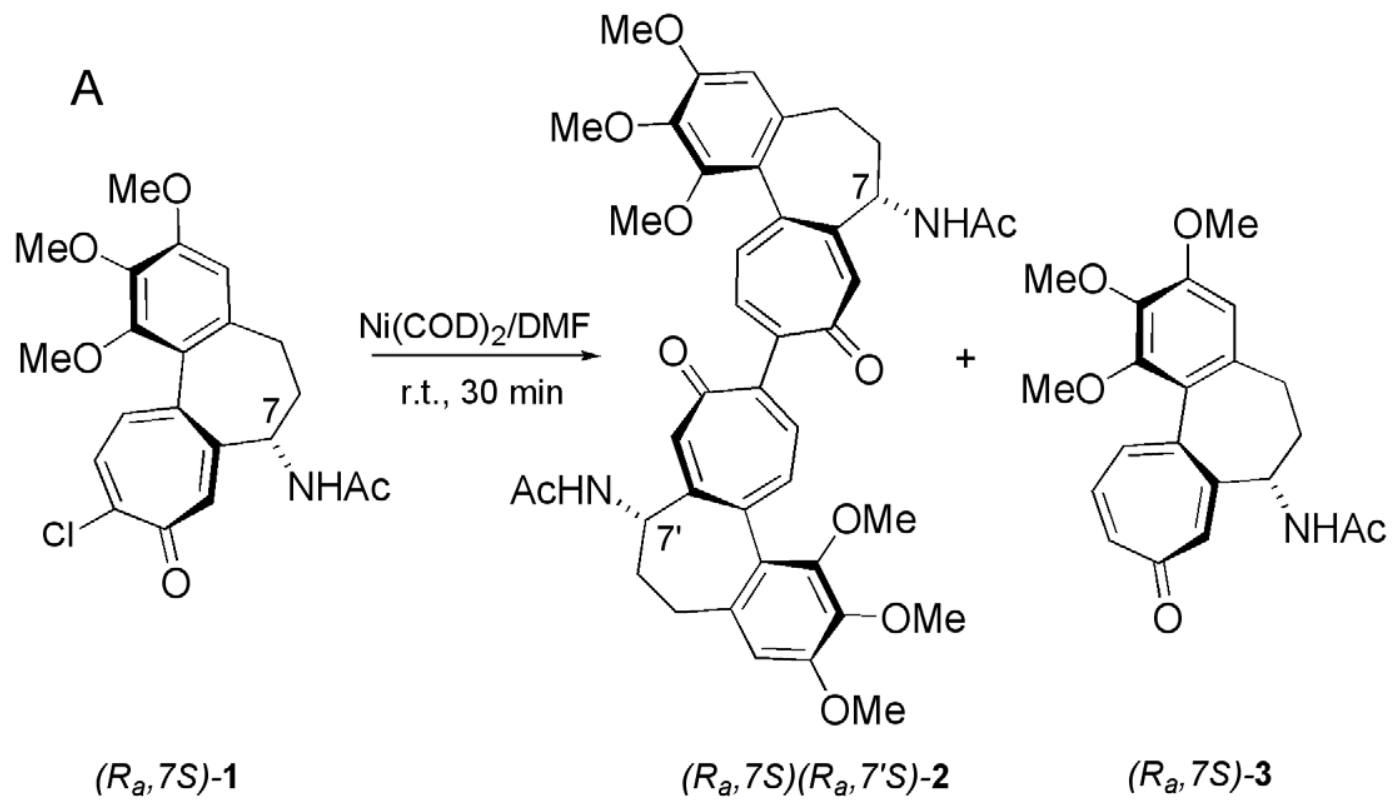

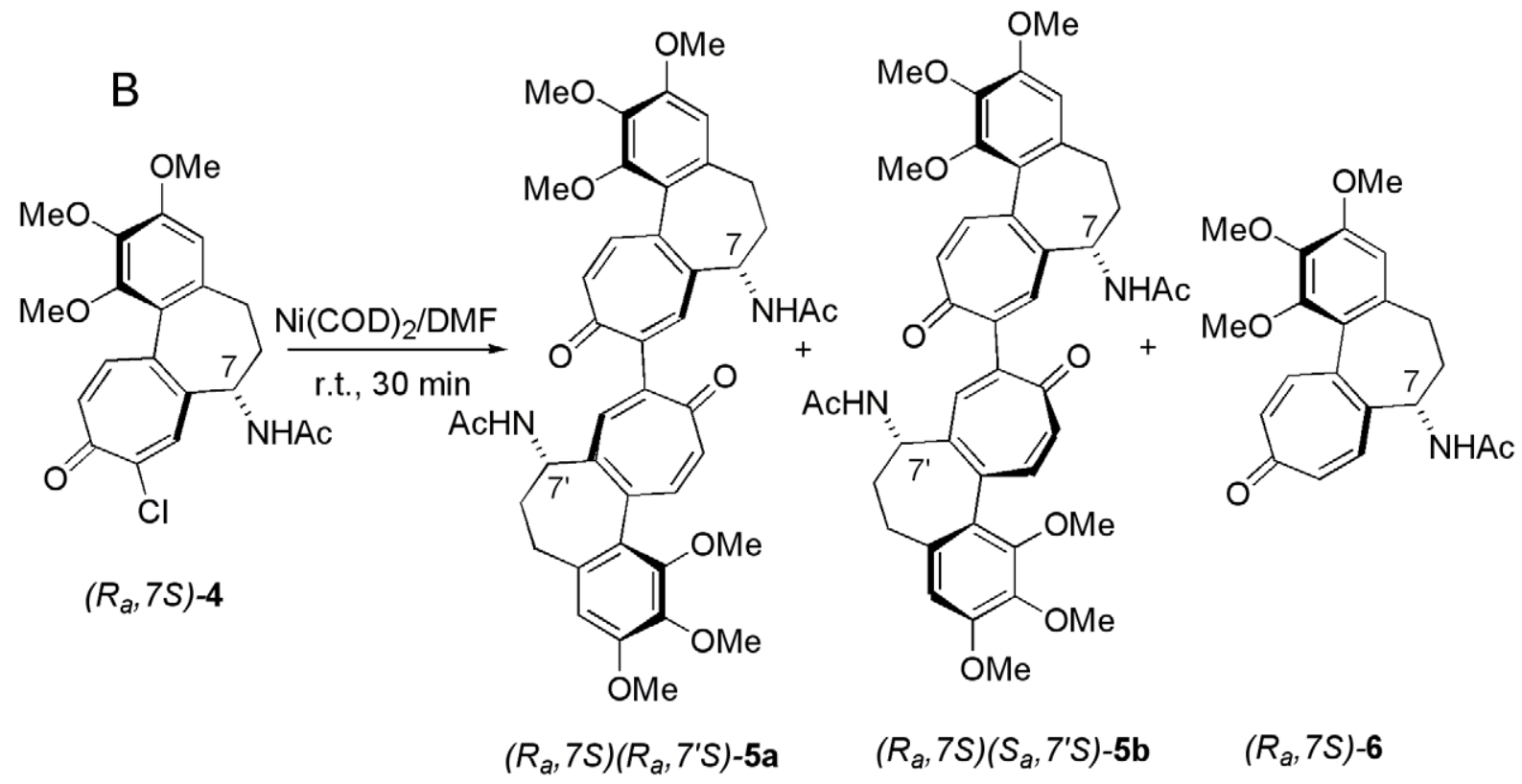

Figure 1. Synthesis of bicolchicide and biisocolchicide. (A) Synthesis of bicolchicide (2), (B) synthesis of biisocolchicide (5) according to the Semmelhack's method. The stereochemical representations of reagents and products are given. doi:10.1371/journal.pone.0010617.g001

DMF at room temperature [9]. This method is further described in Method S1. As shown in Figure 1A for 10-chlorocolchicide (1) [10] and Figure 1B for 9-chlorocolchicide (4) [10], formation of $\mathbf{2}$ from the former, and $\mathbf{5}$ from the latter, was accompanied by hydrogenolysis products, colchicide (3) [11] and isocolchicide (6) [11], respectively. All efforts to carry out these reactions under catalytic conditions with $\mathrm{Ni}(\mathrm{COD})_{2}$ were frustrated. The ${ }^{1} \mathrm{H} \mathrm{NMR}$ ddd pattern for $\mathrm{H} 7$ and $\mathrm{H} 7$ ' supports pseudoaxial assignment for both protons. Since the synthesis started from $(7 S)-\mathbf{1}$, this establishes configuration $\left(R_{\mathrm{a}}, 7 S\right)\left(R_{\mathrm{a}}, 7^{\prime} S\right)$ for compound 2 [12], [13]. For compound 5, ${ }^{1} \mathrm{H} \mathrm{NMR}$ spectra at $600 \mathrm{MHz}$ in $\mathrm{CDCl}_{3}$ at r.t. revealed that $\left(R_{\mathrm{a}}, 7 S\right),\left(R_{\mathrm{a}}, 7^{\prime} S\right)$-biisocolchicide $(\mathbf{5 a})$ and $\left(R_{\mathrm{a}}, 7 S\right)$,
$\left(S_{\mathrm{a}}, 7{ }^{\prime} S\right)$-biisocolchicide $(\mathbf{5 b})$ equilibrate (83\% vs $17 \%$, respectively). These, and all other stereochemical features of these compounds, are dealt with below, step by step, along with spectral evidences.

\section{Discussion}

UV and CD spectral behavior of bicolchicides and biisocolchicides

Figure 2 shows UV and CD spectra in various solvents for the coupling product $\mathbf{2}$ in comparison with its building block, the hydrogenolysis product $\mathbf{3}$. It is seen that the UV and CD bands for 2 , in the low- and medium-energy spectral range $(\lambda>300 \mathrm{~nm})$, 

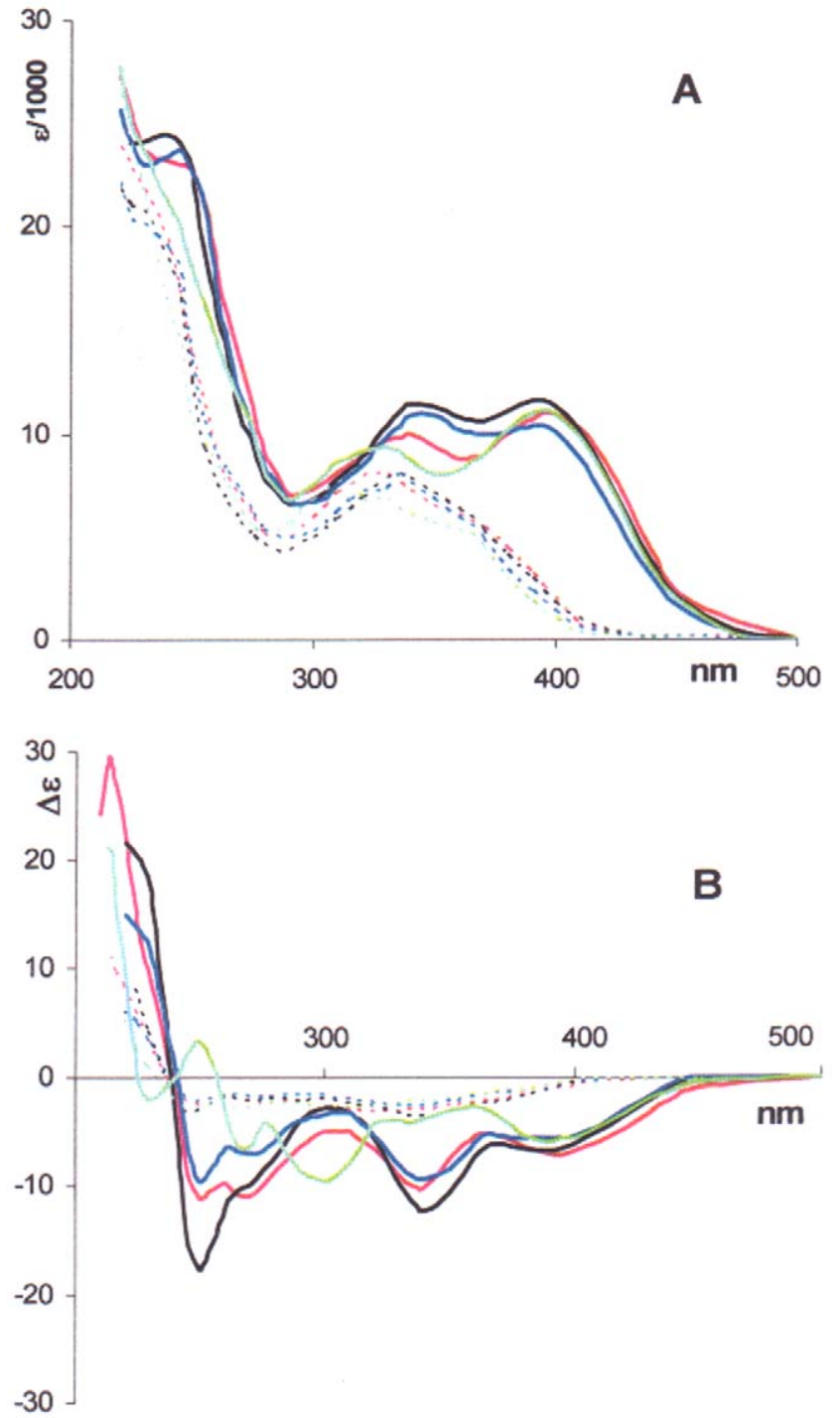

Figure 2. UV and CD spectra for colchicide and bicolchcide. UV (A) and CD (B) spectra for bicolchicide 2 (solid line) and colchicide $\mathbf{3}$ (dotted line) in various solvents are reported; - : $\mathrm{EtOH}_{;} \longrightarrow$ : TFE; $\square$ : $\mathrm{H}_{2} \mathrm{O}$; $-\mathrm{MeCN}$.

doi:10.1371/journal.pone.0010617.g002

undergo a red shift by $c a$. $50 \mathrm{~nm}$ with respect to $\mathbf{3}$, likely a consequence of conjugation between the two moieties, while gaining in complexity. Extensive conjugation in the dimeric species $\mathbf{2}$ and $\mathbf{5}$ is supported by their reduction potential being less cathodic than for the monomeric species $\mathbf{3}$ and $\mathbf{6}$, as determined by cyclic voltammetry. Thus, in dried DMF as solvent, vs SCE, cathodic reduction potentials for compounds $\mathbf{2}$ and $\mathbf{5}$ turned out to be -1.28 and $-1.27 \mathrm{~V}$, against -1.48 and $-1.50 \mathrm{~V}$ for compounds $\mathbf{3}$ and $\mathbf{6}$, respectively. (See Method S2.)

Although the CD bands in the low and medium energy spectral region for both $\mathbf{2}$ and $\mathbf{3}$ have the same (negative) sign, they differ vastly in both shape and intensity. Notably, $\mathbf{2}$ is far more sensitive to the nature of the solvent medium than $\mathbf{3}$. In the higher energy region $(\lambda<300 \mathrm{~nm})$, the intensity of the CD bands of $\mathbf{2}$ is even more deeply modulated by the solvent. Thus, with 2,2,2trifluoroethanol (TFE) and EtOH as solvents, on decreasing the wavelength, the $\mathrm{CD}$ of compound $\mathbf{2}$ attains strongly negative minima, changes its sign at about $240 \mathrm{~nm}$, and acquires positive values at higher energies. In this scenario, the negative and positive CD bands could be considered as branches of an incompletely measured negative exciton couplet. No CD couplet could be observed for $\mathbf{3}$.

With both the coupling product 5 (which, as demonstrated below, exists as an equilibrium mixture of two atropisomers $\mathbf{5 a}$ and $\mathbf{5 b}$, where $\mathbf{5 a}$ highly dominates) and the hydrogenolysis product 6, a similar trend is observed in the low- and mediumenergy spectral range only (Figure 3). Below $300 \mathrm{~nm}$ a positive couplet-like band shows up in alcoholic media like EtOH and TFE. In MeCN and water, the couplet-like nature of the $\mathrm{CD}$ band is somewhat blurred, while in $\mathrm{CHCl}_{3}$ and DMSO recording of $\mathrm{CD}$ spectra was limited to $240 \mathrm{~nm}$ due to strong absorption by the solvents. Remarkably again, the CD with $\mathbf{5}$ proved far more solvent dependent than with 6 .

Such solvent-induced dramatic changes in the GD bands of the coupling products prompted us to investigate in detail the dependence of the spectral changes from the percentage of MeCN in $\mathrm{H}_{2} \mathrm{O}$, keeping in mind that the former is a non hydrogen
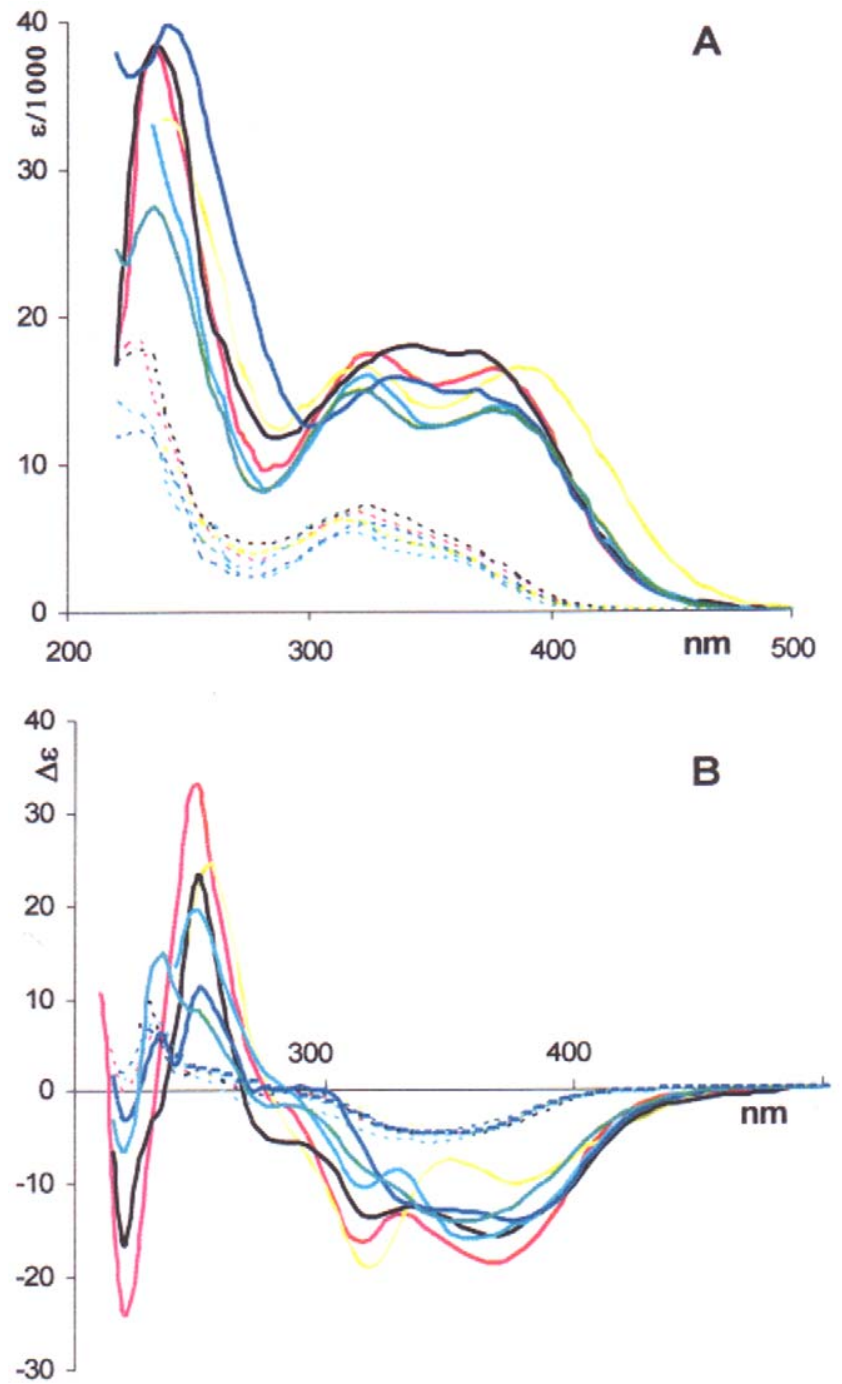

Figure 3. UV and CD spectra for isocolchicide and biisocolchcide. UV (A) and CD (B) spectra for biisocolchicide $\mathbf{5}$ (solid line) and isocolchicide $\mathbf{6}$ (dotted line) in various solvents are reported; - $-\mathrm{EtOH}$; $\longrightarrow$ TFE; $\longrightarrow$ : $\mathrm{H}_{2} \mathrm{O} ; \longrightarrow$ : $\mathrm{MeCN} ; \longrightarrow \mathrm{CHCl}_{3} ; \longrightarrow$ : DMSO.

doi:10.1371/journal.pone.0010617.g003 
bonding, polar solvent, while the latter is strongly polar, hydrogen bonding [14]. On mixing these wholly miscible solvents, a contraction of volume occurs, in an endothermic process. Out of the plethora of contrasting interpretations or models introduced to explain particular experimental data [14], this process can be simply interpreted as a collapse of some "icebergs" arising from strong hydrogen bonding between water molecules. CD spectra of 5 at constant concentration in $\mathrm{MeCN} / \mathrm{H}_{2} \mathrm{O}$ mixtures of nine different compositions - from neat $\mathrm{MeCN}$ to neat $\mathrm{H}_{2} \mathrm{O}$ - are shown in Figure 4. These spectral modifications cannot be attributed to any change in the $[\mathbf{5 a}] /[\mathbf{5 b}]$ atropisomeric equilibrium. Actually, we have proven that the $[\mathbf{5 a}] /[\mathbf{5 b}]$ atropisomeric equilibrium is nearly invariant to all compositions of these solvent mixtures, from neat $\mathrm{H}_{2} \mathrm{O}$ to neat MeCN: HPLC analysis (see Materials and Methods section) of the above mixtures shows that the percentage of $\mathbf{5 a}$ in the atropisomeric mixture remains in the range $83-87 \%$ throughout. In the light of these observations, the nine dichroic absorption curves in Figure 4 reveal the presence of two isodichroic points at 320 and $280 \mathrm{~nm}$ and seven peaks ("extrema") that could be localized, at least in some recorded CD spectra, at 377, 342, 305, 270, 252, 235, and $222 \mathrm{~nm}$. The presence of isodichroic points, for solutions at constant molarity of the solute, is taken as an indication that the actual spectra derive from a combination of basic spectra having an identical value at the isodichroic points. Quantitative analysis of the data reported in Figure 4, from neat $\mathrm{H}_{2} \mathrm{O}$ to neat $\mathrm{MeCN}$, revealed that the measured CD spectra cannot be expressed as a linear combination of two CD spectra, the one in neat water and the other one in neat MeGN.

The consequences of changing the percentage of $\mathrm{H}_{2} \mathrm{O}$ in MeCN can be analyzed by considering the intensity of the $\mathrm{CD}$ signal at the wavelengths indicated above. This is shown in Figure $5 \mathrm{~A}$ and $5 \mathrm{~B}$, where the dichroic elongations $(\mathrm{mm})$ are given as a function of the mole fraction of $\mathrm{H}_{2} \mathrm{O}$. The dichroic curves can be grouped into two families. One includes the stronger bands centered at 377, 342 and $235 \mathrm{~nm}$ (Figure 5A), where no change in the sign of dichroic bands is observed. In this first family of curves, a very swift change of elongation occurs at vanishingly small

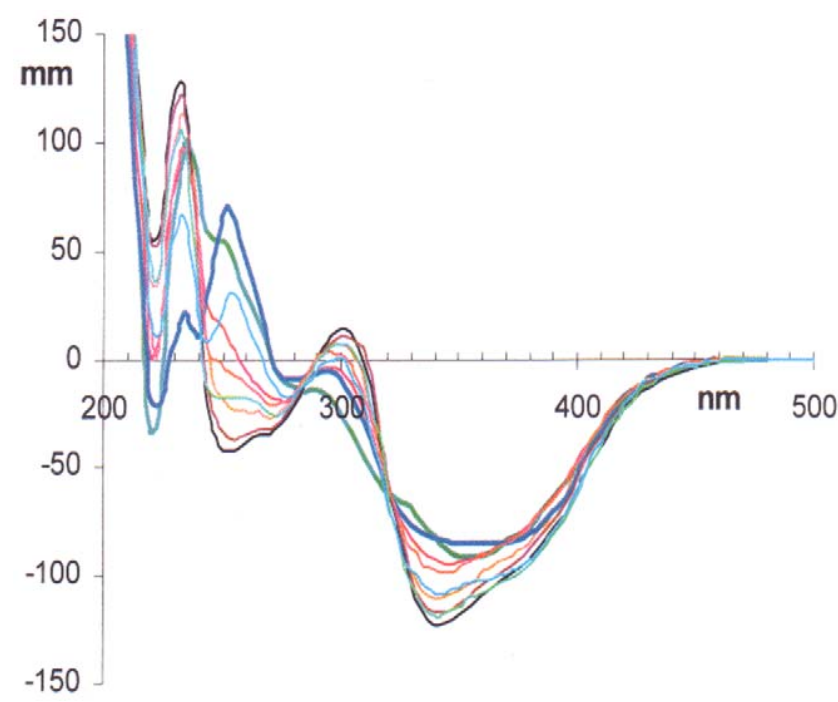

Figure 4. CD spectra for biisocolchicide in $\mathrm{MeCN} / \mathrm{H}_{2} \mathrm{O}$ mixtures of various compositions. $\mathrm{CD}$ spectra for biisocolchicide $\mathbf{5}$ in MeCN/ $\mathrm{H}_{2} \mathrm{O}$ mixtures of various composition are reported; - : neat $\mathrm{H}_{2} \mathrm{O}$; neat $\mathrm{MeCN}$ (bold lines); elongations are in $\mathrm{mm}$.

doi:10.1371/journal.pone.0010617.g004
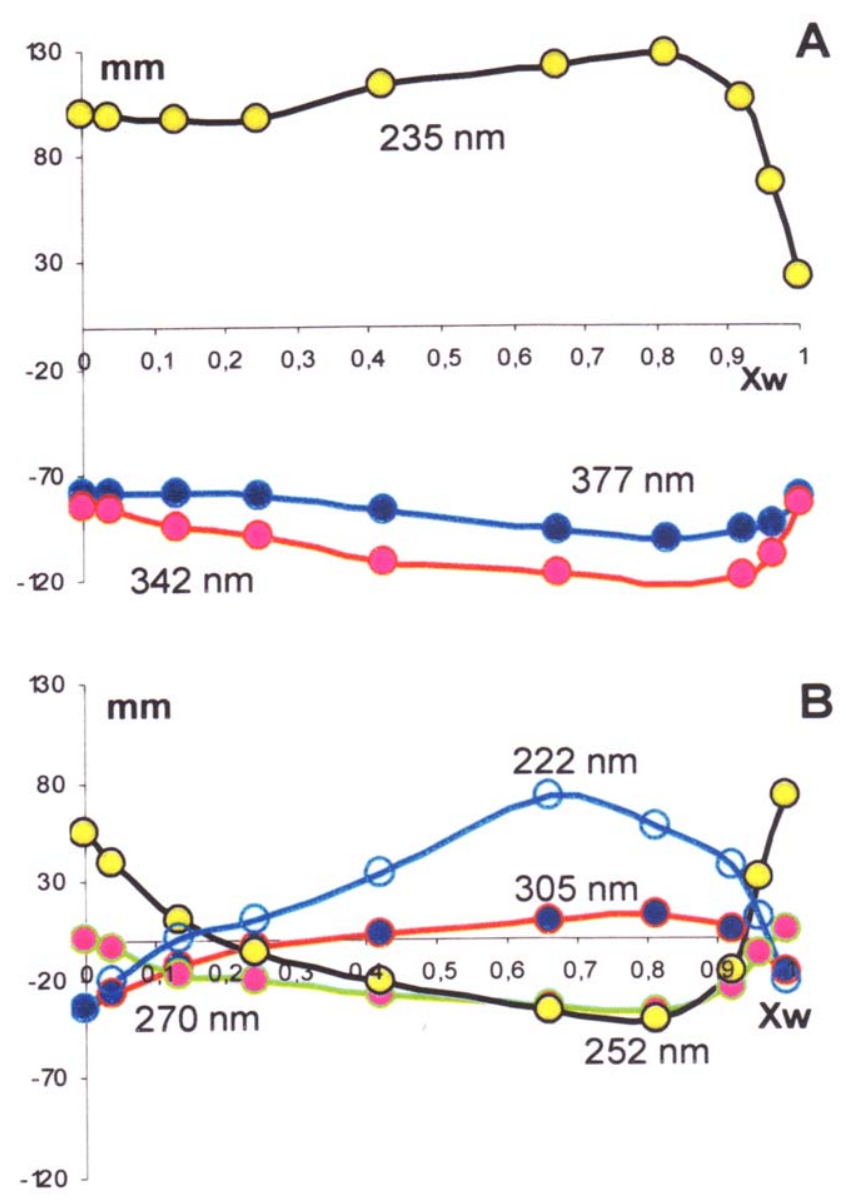

Figure 5. Elongations of the dichroic bands of biisocolchicide with respect to the mole fraction of water. The $C D$ elongations $(\mathrm{mm})$ of 377, 342 and 235 (A) and 305, 270, 252 and $222 \mathrm{~nm}$ (B) dichroic bands, derived from the spectra in Figure 4, with respect to the mole fraction of water are reported.

doi:10.1371/journal.pone.0010617.g005

concentrations of MeCN, where also thermodynamic $\Delta \mathrm{H}$ data revealed some peculiarity of the $\mathrm{MeCN} / \mathrm{H}_{2} \mathrm{O}$ system [15], whilst further additions of MeCN - up to neat MeCN - have relatively scarce effect on the elongations. Such changes in the elongation probably stem from both the well known disruption ability of $\mathrm{OH}$... O hydrogen bonds and the high affinity for the lipophilic parts of the solute by the solvent MeCN. In this scenario, we envisage the presence of two families of conformers, one related to hydrogen bonded structures $\left(\mathrm{X}_{\mathrm{w}} \gg 0.9\right.$ in Figure $5 \mathrm{~A}$, at $\mathrm{X}_{\mathrm{w}}=1$ ) and the other one unrelated to hydrogen bonding $\left(\mathrm{Xw}_{\mathrm{w}}<0.9\right)$. Associating explicit molecular geometries to these frames is a hard task, however, probably beyond the capability of current continuum solvent models. All groups containing oxygen are first candidates for hydrogen bonding. Included are the methoxy groups (in particular the central one in the 1,2,3-trimethoxybenzene moiety, which is significantly more hydrophilic because it is forced out of the plane of the arene ring [15]), the acetylamino groups, and the carbonyl groups.

The negative bands at 377 and $342 \mathrm{~nm}$ must arise from partially conjugated electrons of the two cycloheptatrienone moieties, while the $235 \mathrm{~nm}$ band can be interpreted as the positive branch of a partially measured CD couplet. All that must stem from identical aromatic transitions, each one localized in the trimethoxybenzene moiety and coupled to each other according to the known exciton 
coupling mechanism (see next DeVoe's model section). In both cases, groups such as methoxy (235 nm band) and carbonyl (377 and $342 \mathrm{~nm}$ bands) are present in the moiety which is most involved in the transition, hence most strongly influenced by hydrogen bonding.

The conceivable hypothesis of two structural arrangements, one at $\mathrm{Xw}_{\mathrm{w}}=1$ and a second one at $\mathrm{X}_{\mathrm{MeCN}}=1$, from which the stronger CD bands centered at 377, 342 and $235 \mathrm{~nm}$ originate, does not find a parallel in the analysis of the second family of curves, the weaker dichroic bands at 305, 270, 252 and $222 \mathrm{~nm}$. These, also in the range $\mathrm{Xw}_{w}<0.9$, on changing the composition of the solvent undergo marked changes, including the sign of the bands, as it can be appreciated from Figure 5B. More subtly, we are faced by two contrasting observations with these weaker bands. From one side, slight additions of MeCN to aqueous solutions of compound $\mathbf{5}$ result in steep changes in the elongations, en route to inverting the sign of the bands. From the other side, on adding $\mathrm{H}_{2} \mathrm{O}$ to MeCN solutions of compound $\mathbf{5}$, a more gradual change in the elongations is observed in comparison with what occurs when $\mathrm{X}_{\mathrm{w}}$ is close to 1 . As above, the sign of the bands is reversed. This is a more complex trend than was observed with the stronger bands in Figure 5A, where at each wavelength-band two limiting bands were observed on changing from neat MeCN to neat $\mathrm{H}_{2} \mathrm{O}$. Taking into account the low intensity, the close values of frequency with respect to other intense bands, and the complex mechanisms that exist (vide infra) for a transfer of rotational strength from one band to another one, no firm conclusion can be reached at this time about the mechanism underlying the spectral changes with these minor $\mathrm{CD}$ bands.

\section{DeVoe's mechanisms for the origin of circular dichroism from asymmetric locations of chromophores}

The onset of exciton couplets suggests that the phenomena described in the previous section are amenable to a rationalization on the basis of DeVoe's classical model [3], [4]. This model is particularly well suited to treat "dimeric" molecules where, like with $\mathbf{2}$ and $\mathbf{5}$, chirality is also contributed by deviations from coplanarity of a couple of identical, or nearly identical, quasiplanar chromophores, e.g. the trimethoxy-benzene chromophores. The model can also account for the high sensitivity of CD spectra to relatively minor conformational variations, as it is observed with coupling products $\mathbf{2}$ and $\mathbf{5}$, from what is likely a solvent effect.

Equation (1), which was derived from a coupled oscillator approach [6], expresses the CD due to a series of transitions $1,2,3 \ldots$ i localized in the chromophores of the molecule and coupled through their dipole-dipole interactions. In this frame, transitions related to strongly conjugated electrons, such as those involved in the $\lambda>300 \mathrm{~nm}$ spectral range, cannot be considered.

Equation (1), under simplifying physical conditions, can be used to discuss the higher-energy transitions $(\lambda<300 \mathrm{~nm})$ thanks to an explicit expression of the dependency of $\Delta \varepsilon$ from both the molecular geometry and the UV spectral features of $\mathbf{1}$ and $\mathbf{4}$ :

$$
\Delta \varepsilon=0.0073 \pi^{2} \mathrm{~N} v^{2} \sum_{i>j} \mathbf{e}_{\mathrm{i}} \times \mathbf{e}_{\mathrm{j}} \cdot \mathbf{R}_{\mathrm{ij}} \operatorname{Im} \mathrm{A}_{\mathrm{ij}}
$$

Here, Im Aij is the imaginary part of a generic element of matrix $\mathrm{A}$, while $\mathrm{A}$ is the matrix inverse of matrix $\mathrm{B}$, whose generic element $B_{i j}$ is defined by $B_{i j}=\delta_{i j} / \alpha_{i}+G_{i j}$. In this definition, $\alpha_{i}$ is the electric polarizability allied to the $\mathrm{i}^{\text {th }}$ electronic transition, while $\mathrm{G}_{\mathrm{ij}}$ (Equation (2)) is the interaction energy between two unit pointdipoles $\mathbf{e}_{\mathrm{i}}$ and $\mathbf{e}_{\mathrm{j}}$. These represent the orientation features of dipole transitions $\mathrm{i}$ and $\mathrm{j}$, which are localized in different points of the molecule and are interconnected by the distance $\mathbf{R}_{\mathrm{ij}}$.

$$
\mathrm{G}_{\mathrm{ij}}=\frac{\mathbf{e}_{\mathrm{i}} \cdot \mathbf{e}_{\mathrm{j}}}{\mathrm{R}_{\mathrm{ij}}^{3}}-\frac{3 \mathbf{e}_{\mathrm{i}} \cdot \mathbf{R}_{\mathrm{ij}} \mathbf{e}_{\mathrm{j}} \cdot \mathbf{R}_{\mathrm{ij}}}{\mathrm{R}_{\mathrm{ij}}^{5}}
$$

Because the polarizability is related to the wavelength, matrix inversion has to be made at each value of $\lambda$.

A qualitative analysis can be easily carried out under further simplifying conditions, as embodied in Equation (3), which is derived from Equation (1). Equation (3) holds for two identical chromophores with one electrically-allowed transition, in the frame of a treatment first order in the dipole-dipole $\mathrm{G}_{12}$ term [6].

$$
\Delta \varepsilon(v)=\mathrm{Ce}_{1} \times \mathbf{e}_{2} \cdot \mathbf{R}_{12} \mathrm{G}_{12} v^{2} \alpha_{1}^{\prime}(v) \alpha_{1}^{\prime \prime}(v)
$$

Here, $\mathrm{C}$ is a constant, $\alpha_{1}{ }^{\prime}$ and $\alpha_{1}{ }^{\prime \prime}$ are the real and the imaginary parts of polarizability $\alpha_{1}$ pertaining to the transition " 1 ", and $\mathbf{R}_{12}$ is the distance between the two dipoles corresponding to the same electronic transition localized in the two molecular moieties.

Two notable features emerge from Equation (3) for the CD generated at the transition " 1 " spectral range. The first feature is the typical spectral shape of the couplet, which is due to the product of the imaginary part of the polarizability (the $\alpha_{1}{ }^{\prime \prime}(v)$ term) (which acquires maximum value at the wavelength of maximum absorption, $\left.\lambda_{\max }\right)$ and the real part of the polarizability (the $\alpha_{1}{ }^{\prime}(v)$ term). The latter, for gaussian or lorentzian spectral shape of $\alpha_{1}{ }^{\prime \prime}(v)$, undergoes inversion of sign at $\lambda_{\max }$, from positive values at $\lambda>\lambda_{\max }$, to negative values at $\lambda<\lambda_{\max }$, following an asymmetric trend with respect to the $\lambda_{\max }$ position. The second feature that emerges from Equation (3) is that the sign and intensity of $\Delta \varepsilon$ depend on the molecular geometry, both through the triple mix product of the three vectors that identify the asymmetry of the position/orientation of transition dipoles in the molecule $\left(\mathbf{e}_{1}, \mathbf{e}_{2}\right.$, $\mathbf{R}_{12}$ ) and the scalar products of vectors present in the energy term $\mathrm{G}_{12}$. Thus, rotation of the two moieties in products $\mathbf{2}$ and $\mathbf{5}$ away from a common plane, where $\Delta \varepsilon=0$, gives rise to a positive or a negative couplet; the rate of increase of intensity with the deformation parameter turns out to be proportional to both the square of the molar absorption coefficient of the two interacting identical transitions and the dipolar interaction terms. This means that only strong absorption bands can emerge in the CD spectrum with the typical couplet shape. The $\mathrm{R}_{12}$ distance between the trimethoxy-benzene moieties is also relevant. It is as high as 14.5 or $13.7 \AA$ for compounds $\mathbf{5 a}$ and $\mathbf{5 b}$, respectively, in their most stable conformation, as indicated by DFT minimizations (see the computational section). Thus, because of a $\left(1 / R_{12}\right)^{3}$ dependence of $\mathrm{G}_{12}$, it is only with particularly favourable orientations of the above three vectors $\left(\mathbf{e}_{1}, \mathbf{e}_{2}, \mathbf{R}_{12}\right)$ that the couplet could emerge.

In the presence of a second transition " 2 ", not overlapping the one in Equation (3), a term expressed by Equation (4) adds to $\Delta \varepsilon$ from the couplet [7].

$$
\Delta \varepsilon(\lambda)=\operatorname{Dv} \varepsilon_{1}(\lambda) \mathbf{e}_{1} \times \mathbf{e}_{2} \cdot \mathbf{R}_{12} \mathrm{G}_{12} \alpha_{2}^{\prime \prime}(\lambda)
$$

In Equation (4), D is a constant and " 1 " represents quantities related to the above described electrically-allowed transition confined to one of the molecular moieties that give rise to the couplet. In turn, "2" represents quantities related to any other transition that does not overlap transition " 1 " and which is confined to the other molecular moiety. In this case - geometric 
factor $\mathrm{e}_{1} \times \mathrm{e}_{2} \mathrm{R}_{12}$ apart - the contribution of transition " 2 " to the $\mathrm{CD}$ in the spectral zone of transition " 1 " is proportional to the molar coefficient $\varepsilon_{1}(\lambda)$ and is positive or negative for absorption band " 2 " lying at shorter or longer wavelength with respect to band " 1 ". Terms of this type may induce a shift of the couplet towards positive or negative values, while also causing a deformation of the couplet itself. Joint action of multiple effects of this type can make very difficult to even recognize the presence of a couplet. Probably multiple effects of this type are at work with compounds $\mathbf{2}$ and 5, particularly in the case of the bands of Figure $5 \mathrm{~B}$, which further complicates the analysis of these bands. When other transitions overlap, even partially, transition "1", difficulties of interpretation reach the apex.

\section{Stereochemical assignments of bicolchicide 2 and biisocolchicide 5 , and mutarotation with the minor conformer $5 b$}

Other than in the opposite sign of the couplet in the high-energy CD spectral region - as said above - coupling products $\mathbf{2}$ and $\mathbf{5}$ differ dramatically in both HPLC chromatographic and ${ }^{1} \mathrm{H}$ NMR spectral behavior. While compound $\mathbf{2}$ showed up, under all circumstances, as a single conformer, the ${ }^{1} \mathrm{H}$ NMR spectra of compound $\mathbf{5}$ in $\mathrm{CDCl}_{3}$, at either 300 or $600 \mathrm{MHz}$, could only be interpreted by disentangling signals for two conformers, $\mathbf{5 a}$ and $\mathbf{5 b}$ (Figure 1), in a 4.8:1 peak-area ratio. As HPLC analyses of the mixture gave a similar peak-area ratio for $\mathbf{5 a}$ vs $\mathbf{5 b}$ (Table 1 and Materials and Methods), the analysis in other solvents was economically carried out from HPLC data alone. The results in Table 1 fail to reveal any trend in the $\mathbf{5 a / 5 b}$ population ratio with the bulk properties of the medium.

While $\left(R_{a}, 7 S\right)\left(R_{\mathrm{a}}, 7^{\prime} S\right)$ configurational attribution to $\mathbf{2}$ and $\mathbf{5 a}$ is based on ${ }^{1} \mathrm{H}$ NMR spectra alone (ddd pattern for $\mathrm{H} 7$ and $\mathrm{H} 7$ ' for both $\mathbf{2}$ and 5a, see Materials and Methods), the stereochemical assignment of the minor conformer $\mathbf{5 b}$ was first carried out on the basis of CD spectra. To do so, mixtures containing compound $\mathbf{5}$ were subjected to HPLC, peak eluates being directly collected into thermostatted $\mathrm{CD}$ cuvettes at $c a 1^{\circ} \mathrm{C}$ and rapidly $\mathrm{UV}$ and $\mathrm{CD}$ analyzed. While the UV spectra proved very similar for all eluates, the dichroism for a minor peak eluted at $t_{\mathrm{R}}=8.07 \mathrm{~min}$ turned out to increase with time, extrapolating at infinite time $(c a .24 \mathrm{~h})$ to the dichroism observed for the major peak $\left(t_{R}=6.62 \mathrm{~min}\right)$, as shown in Figure 6. Initially, the dichroism in the area centered at $\lambda=350$ $\mathrm{nm}$ was only weakly negative, with elongation much smaller than at infinite time. This is consistent with a partial compensation from the interconnected opposite helices $\left(R_{\mathrm{a}}, 7 S\right)\left(S_{\mathrm{a}}, 7^{\prime} S\right)$.

Table 1. Experimental distribution of $5 \mathrm{a}$ and $5 \mathrm{~b}$ atropisomers at $300 \mathrm{~K}$.

\begin{tabular}{lllll}
\hline & & & & \\
\hline Method & Medium & $\varepsilon^{\text {[a] }}$ & $\mathbf{5 b / 5 a}$ & $\Delta \mathbf{G}_{\mathbf{3 0 0}}(\mathbf{K c a l} / \mathbf{m o l})$ \\
\hline${ }^{1} \mathrm{H}$ NMR or HPLC & $\mathrm{CDCl}_{3}$ & 4.7 & $17 / 83$ & 0.8 \\
HPLC & $\mathrm{C}_{2} \mathrm{H}_{5} \mathrm{OH}$ & 24.3 & $5 / 95$ & 1.7 \\
$\mathrm{HPLC}$ & $\mathrm{CF}_{3} \mathrm{CH}_{2} \mathrm{OH}$ & 26.5 & $22 / 78$ & 0.8 \\
$\mathrm{HPLC}$ & $\mathrm{CH}_{3} \mathrm{OH}$ & 32.6 & $6 / 94$ & 1.7 \\
$\mathrm{HPLC}$ & $\mathrm{CH}_{3} \mathrm{CN}$ & 36.2 & $13 / 87$ & 1.3 \\
$\mathrm{HPLC}$ & $\left(\mathrm{CH}_{3}\right)_{2} \mathrm{SO}$ & 49 & $3 / 97$ & 2.2 \\
$\mathrm{HPLC}$ & $\mathrm{H}_{2} \mathrm{O}$ & 78.5 & $17 / 83$ & 0.8 \\
\hline
\end{tabular}

[a] Dielectric constant.

doi:10.1371/journal.pone.0010617.t001
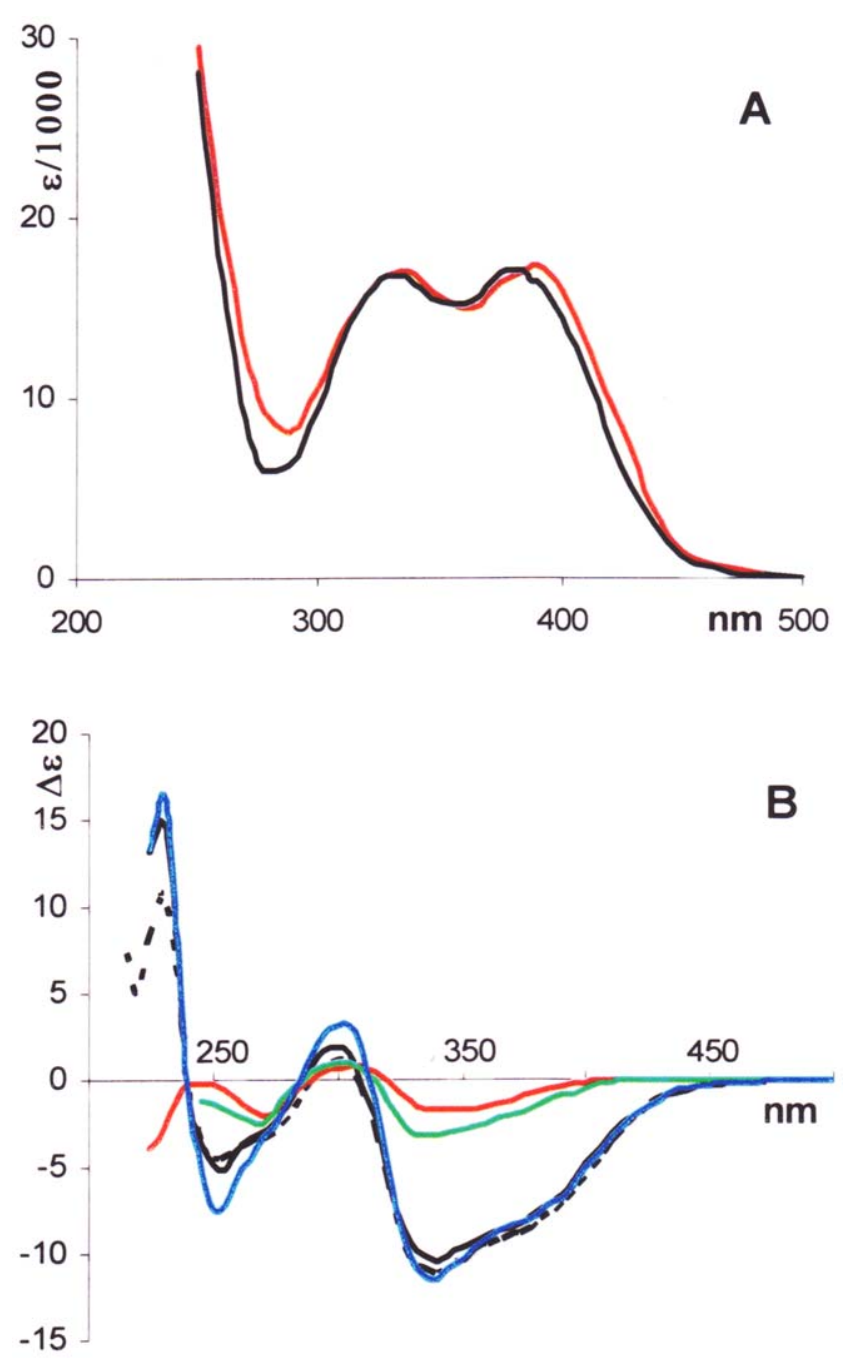

Figure 6. HPLC separation of conformers $5 \mathrm{a}$ and $\mathbf{5 b}$. The Figure shows: (A) UV spectra in $\mathrm{MeCN} / \mathrm{H}_{2} \mathrm{O} 40: 60$ for $\mathbf{5 a}(-)$ and $\mathbf{5 b}(-)$. (B) $\mathrm{CD}$ spectra at ca $1^{\circ} \mathrm{C}$ for $\mathbf{5 a}(-)$ and $\mathbf{5 b}$ after 6-30 $\mathrm{min}(-)$; $95-120$ min (-); overnight $(-)$; finally $C D$ spectrum for the equilibrium mixture $\mathbf{5 a / 5 b}$ at r.t. (-----).

doi:10.1371/journal.pone.0010617.g006

Configurational assignment $\left(R_{\mathrm{a}}, 7 S\right)\left(S_{\mathrm{a}}, 7^{\prime} S\right)$ to $\mathbf{5 b}$ was confirmed from ${ }^{1} \mathrm{H}$ NMR spectra in $\mathrm{CDCl}_{3}$, which showed two sets of protons for $\mathrm{H} 7$ and $\mathrm{H} 7$ ', differing both as to the chemical shift and the coupling pattern. A ddd pattern supports pseudoaxial assignment to $\mathrm{H} 7$ (i.e. the $\mathrm{C} 7$ acetamido group takes a pseudoequatorial position) for the $R_{\mathrm{a}}$ part, while a dd pattern supports pseudoequatorial assignment to $\mathrm{H} 7$ ' (i.e. the $\mathrm{C} 7$ acetamido group takes a pseudoaxial position) for the $S_{\text {a }}$ part [12], [13].

The time dependency observed for the dichroism of compound 5 must stem from mutarotation, with the minor conformer $\left(R_{\mathrm{a}}, 7 S\right)\left(S_{\mathrm{a}}, 7 ' S\right)-\mathbf{5 b}$ undergoing helical inversion to give rise to $\left(R_{\mathrm{a}}, 7 S\right)\left(R_{\mathrm{a}}, 7^{\prime} S\right)-\mathbf{5 a}$. The equilibrium constant $\mathrm{K}_{(\boldsymbol{S a})-\mathbf{5} /(\boldsymbol{R a})-5}$ between the two atropisomers is sensitive to the solvent nature (Table 1), and, like for the CD spectra of compounds $\mathbf{2}$ and $\mathbf{5}$, the changes can hardly be related to the bulk properties of the medium. Moreover, the trend observed for the separate moieties (let them be represented by the known colchicine (7) and isocolchicine (8), Figure 7), is also not followed. 
<smiles>CCCCCCCCCN[C@H]1CCc2cc(OC)c(OC)c(OC)c2-c2ccc(OC)c(=O)cc21</smiles>

$\left(R_{a}, 7 S\right)-7$

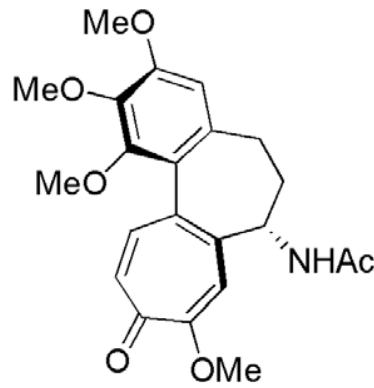

$\left(R_{a}, 7 S\right)-8$

Figure 7. Structure of colchicine and isocolchicine. These structures represent the $\left(R_{\mathrm{a}}, 7 \mathrm{~S}\right)$ configuration of both colchicine (7) and isocolchicine (8).

doi:10.1371/journal.pone.0010617.g007

Rapoport and Lavigne first rationalized the mutarotation of $\mathbf{8}$ in chloroform as due to atropisomeric inversion [16]. They also attributed lack of mutarotation of isocolchicine in ethanol to increased steric bulk of the ethanol-solvated pseudoaxial acetylamino group [16]. Although lack of mutarotation for isocolchicine in ethanol is also consistent with favored solvation of the more exposed pseudoequatorial acetylamino group stabilizing this conformation [17], whichever rationalization applies to this solvent effect, the behavior of the "dimeric" structures, resulting from coupling of either two colchicides, or two isocolchicides, is formally in line with their "monomeric" components. To this concern, it should be noticed that dominance of the $\left(R_{\mathrm{a}}, 7 S\right)\left(R_{\mathrm{a}}, 7^{\prime} S\right)$ $\mathbf{5 a}$ atropisomer by $4.8: 1$ over the $\left(R_{\mathrm{a}}, 7 S\right)\left(S_{\mathrm{a}}, 7, S\right)$-5b atropisomer in $\mathrm{CHCl}_{3}$ at r.t. is less than observed $(10: 1)$ for the $\left(R_{\mathrm{a}}, 7 S\right)$ vs the $\left(S_{\mathrm{a}}, 7 S\right)$ atropisomer of isocolchicine 8 [17]. That is, "dimerization" smoothes out any difference between the equilibrating atropisomers. That said, it should be noticed that the CD spectra of the ensemble (Figure 3B) depend more on solvent effects on conformer 5a than on the position of the equilibrium of this conformer with $\mathbf{5 b}$, in spite of profound differences that exist in the dichroism of the two conformers, $\mathbf{5 a}$ and $\mathbf{5 b}$ (Figure $6 \mathbf{B}$ ).

\section{Computational treatment of bicolchicides and biisocolchicides}

Structure $\mathbf{5}$ was minimized in vacuum by both global space search with molecular mechanics [18] and simulated annealing molecular dynamics [19]. Three types of conformers, $\left(R_{\mathrm{a}}, 7 S\right)\left(R_{\mathrm{a}}, 7^{\prime} S\right)$, $\left(R_{a}, 7 S\right)\left(S_{a}, 7^{\prime} S\right)$, and $\left(S_{a}, 7 S\right)\left(S_{a}, 7^{\prime} S\right)$ emerged. While the latter one is of no interest for its high potential energy, the lowest-energy conformers of the other two types were further minimized by DFT calculations with the M05-2X functional [20] at 6-31G* basis set level (see Calculations S1). The choice of this recent density functional was dictated by its correct treatment - even at this modest basis set level - of the component moieties, colchicine (7) and isocolchicine $(\mathbf{8})$, where the B3LYP density functional resulted in a much too high puckering of the cycloheptatrienone ring [17]. Success of M05-2X could be attributed to a better treatment of medium-range correlations than by B3LYP [20]. As shown in Figure 8 , with both $\left(R_{\mathrm{a}}, 7 S\right)\left(R_{\mathrm{a}}, 7^{\prime} S\right)-\mathbf{5 a}$ and $\left(R_{\mathrm{a}}, 7 S\right)\left(S_{\mathrm{a}}, 7^{\prime} S\right)$-5b the cycloheptatrienone ring was simulated correctly by using the M05$2 \mathrm{X}$ functional, with only slight puckering in accordance with $\mathrm{X}$ ray diffraction data for colchicinoids [21], [22] or nematic-phase NMR for tropone [23]. According to these DFT calculations,
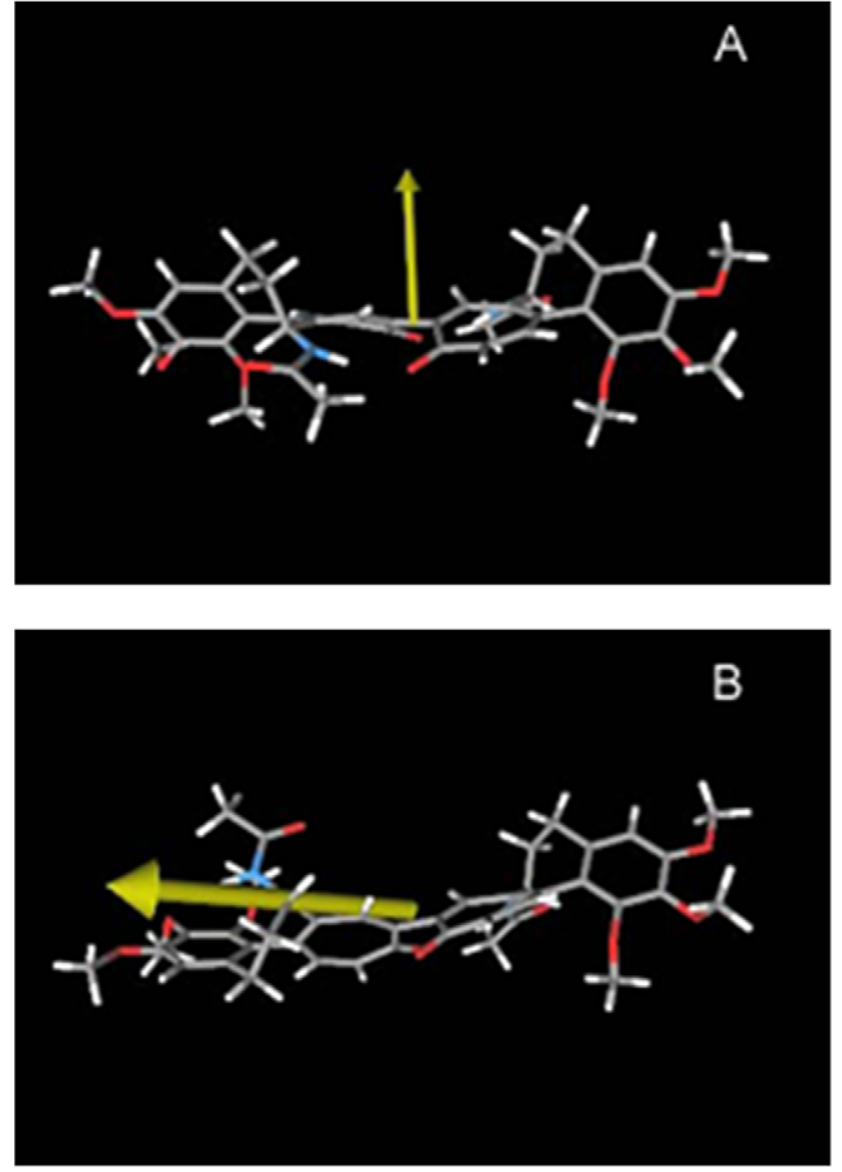

Figure 8. Geometry optimized structures of biisocolchicide atropisomers. The geometry optimized structures (level DFT/M05-2X/ 6-31G*, in vacuum) of biisocolchicide atropisomers $\left(R_{a}, 7 S\right)\left(R_{a}, 7^{\prime} S\right)-5 \mathbf{a}(\mathrm{A})$ and $\left(R_{a}, 7 S\right)\left(S_{a}, 7\right.$ 'S)-5b (B) are shown. The yellow arrow indicates the direction of the dipole moment.

doi:10.1371/journal.pone.0010617.g008

atropisomer $\mathbf{5 a}$ is more stable than $\mathbf{5 b}$ by $2.5 \mathrm{kcal} \mathrm{mol}^{-1}$. Single point MP2 energy calculations at the same set of basis level, which better account for electron correlations, $\mathbf{5 a}$ turned out to be more stable than $\mathbf{5 b}$ by a smaller margin, $1.8 \mathrm{kcal} \mathrm{mol}^{-1}$. Still, this is a larger margin than observed experimentally, with the exception of experiments in DMSO as solvent, where calculated values match experimental values (Table 1). However, in view of the lack of any trend of the experimental energy with the solvent properties (Table 1), the agreement observed for DMSO as solvent should be considered as fortuitous. In view of these facts, the coupling product 5 represents a more challenging system than either $\mathbf{8}$ or $\mathbf{7}$, where the energy predictions by DFT or MP2 in vacuum are in good agreement with the experimental data in $\mathrm{CHCl}_{3}$ as solvent [16]. Likely, this reflects the higher complexity of $\mathbf{5}$.

Quantum mechanical simulation of the UV and CD spectra of atropisomers $\mathbf{5 b}$ and $\mathbf{5 a}$ was also carried out in the frame of the timedependent density functional theory (TDDFT), by using BHLYP, a hybrid functional with a large amount of Fock-exchange [24], with TZVP as basis set. Because the RI-DFT mode was used, the auxiliary basis set TZV/J had to be added. It is seen from Figure 9A for atropisomer $5 \mathbf{a}$ that the low-energy bands only $(\lambda>300)$ were decently reproduced. It can also be noticed that a plotting common artifice, broadening the CD bands with $\sigma=0.32$, seems to afford a better fitting with respect to narrower bands, e.g. $\sigma=0.16$ [25]. 

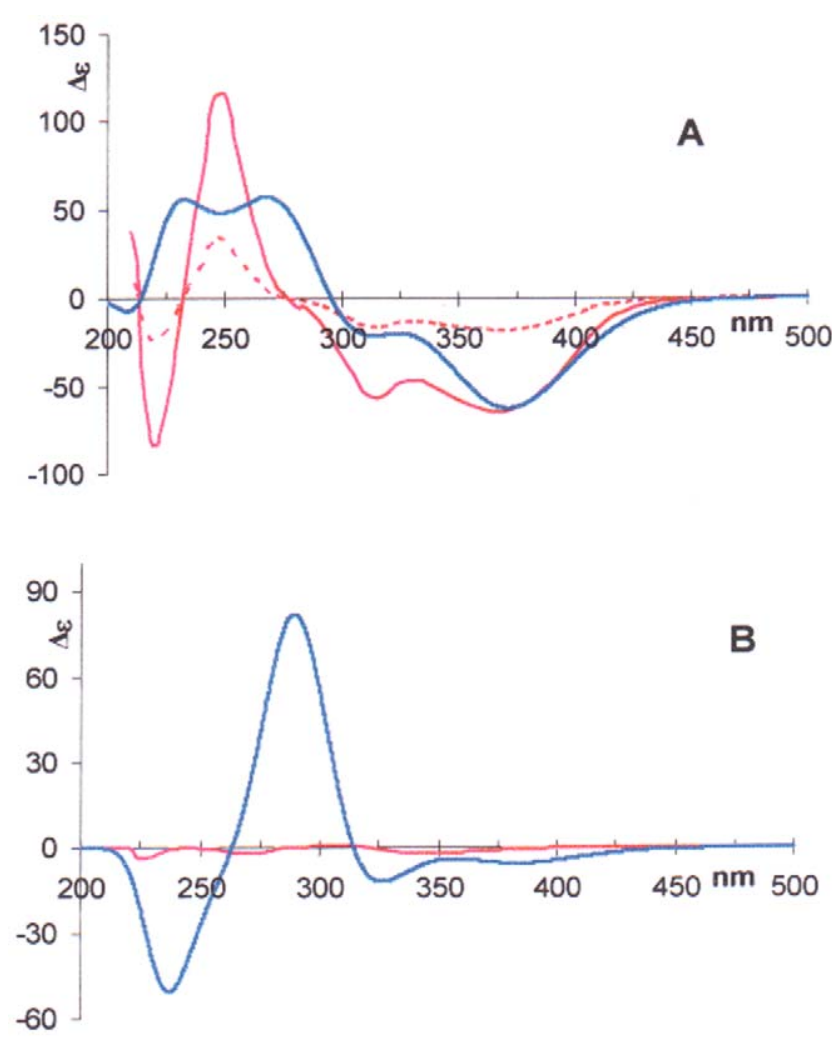

Figure 9. Calculated CD spectra of $\mathbf{5 a}$ and $\mathbf{5 b}$. (A) CD spectra of 5a. - - - : experimental spectrum in $\mathrm{EtOH} ;-$ : the same multiplied by three; - calculated spectrum in $\mathrm{EtOH}, \sigma=0.32$. (B) $\mathrm{CD}$ specta of 5b. - experimental spectrum in $\mathrm{MeCN} / \mathrm{H}_{2} \mathrm{O}$ 40:60; $\longrightarrow$ : calculated spectrum in $\mathrm{EtOH}, \sigma=0.32$.

doi:10.1371/journal.pone.0010617.g009

Actually, the substance of the simulation does not change: the bands at higher energy $(\lambda<300)$ could not be reproduced. With atropisomer $\mathbf{5 b}$, the two moieties interconnected at the cycloheptatrienone rings have opposite helicity (Figure 1B). This leads to only weak (negative) CD (Figure 9B). Our simulations are in accordance for the lowenergy zone $(\lambda>300)$, while, like for $\mathbf{5 a}$, the high-energy zone $(\lambda<300)$ could not be reproduced.

As far as solvent effects are concerned, it must be said that DFT calculations with continuum solvation models, such as devised by Klamt [26], Caricato [27], Cramer and Truhlar[28], and Florián and Waershel [29], do not attain a precision sufficient to deal with the tiny conformational energy differences with atropisomers $\mathbf{5 a}$ and $\mathbf{5 b}$ and therefore were not even considered for present tasks. $\mathrm{MD}$ procedures in explicit solvent could be carried out in a periodic system, also with pseudopotential plane-wave density functional theory, such as implemented in the NWChem suite [30], or in linear-scaling implementations [31], where parameterization is carried out on-the-fly. A limitation to this theory can be seen in the requirement of trajectories long enough to reach convergence, which would be computationally too costly for molecules of the size of bicolchicides. More fundamentally, the currently implemented Becke-Lee-Yang-Parr (BLYP) density functional [31] is inadequate to treat the cycloheptatrienone nucleus, where even the more advanced hybrid functional B3LYP failed [17]. On the other hand, any treatment of biisocolchicide conformers by classical MD would require to build an ad hoc highlevel force field, without any guaranty of success given the tiny energy differences into play.
What remains open to question, are QM-MM calculations, where the solute is treated quantum mechanically, while the solvent is treated classically [32]. A foreseeable problem here is that MD procedures are characterized by extensive sampling in view of long-enough trajectories, in the order of many ns so that, to alleviate the burden of the big matrices, QM-MM procedures of the type required in this work are currently carried out with a semiempirical level for the QM part [32], thus inheriting all limitations of semiempirical treatments. Nonetheless, we tried this route. Thus, PM3 was attempted in explicit solvents with Amber 10 suite and GAFF force field [33], resulting in an unrealistic strong puckering of the cycloheptatrienone rings and unreliable energy output. On the other hand, the SCG-DFTB level [34], attempted in vacuum, led to similarly disappointing results.

\section{Conclusions}

A central observation in this work is that DeVoe's model for circular dichroism [3], [4] is very important. In fact this model allows us to perform a successful qualitative analysis of the dichroic behaviour of both $\mathbf{2}$ and $\mathbf{5}$. For any quantitative application of DeVoe's model [3], [4] prospects are far less alluring, however. In order that the model retains validity in quantitative applications, the electronic transitions on the component moieties of our coupling products should be independent from one another, while with compounds $\mathbf{2}$ an $\mathbf{5}$ it is expected that DeVoe's model is invalidated by substantial conjugation between the component moieties. For the bands at higher energy, in particular those involved in exciton couplets, the number, wavelength features, and polarization direction of the transitions need to be accurately known in order that the CD spectrum can be predicted in the frame of DeVoe's model. This would require a reliable deconvolution of the absorption spectrum for the moieties that constitute the coupling products $\mathbf{2}$ and $\mathbf{5}$ into the component bands, as well as the measurement of polarization directions of related transitions. Moreover, the geometrical relationships between the moieties in the global molecule should be accurately known in view of the sensitivity of the CD to even small changes in the relative orientation of transition dipoles localized at each trimethoxy-benzene moiety. These changes may turn out to be cumulative effects of small distortions along the molecular backbone, of neat significance for equations (1-4).

All that draws attention to four key points: (1) the trimethoxybenzene chromophores are separated by a large distance, ca. $14 \AA$, (2) they are interconnected by flexible moieties that are exposed to the solvent medium, (3) the CD is highly sensitive both to the relative orientation of these chromophores and (4) the many existing transitions which can modify the couplet profile. Thus, CD couplets can only emerge clearly when favorable orientations of the transition dipoles are met, which, unsurprisingly, can only happen for certain solvents. All that should be considered in the above illustrated framework, i.e., that for multichromophoric molecules the interplay of various factors in the interaction with circularly polarized light may face such a delicate balance of factors that the presence of the couplet may even escape attention.

Our limited success in the quantum mechanical simulation of the $\mathrm{CD}$ of atropisomers $\mathbf{5 a}$ and $\mathbf{5 b}$ reflects general obstacles encountered in such computations, whose importance in any real case is difficult to assess. These include basis set non-completeness (which, admittedly, could be ameliorated, albeit at much increasing computational cost), difficulty in thoroughly accounting for electron correlation, zero-point vibrations, and any conceivable tunneling between multiple energy minima for the different conformations that are very close in energy [35]. Unavailability of 
adequate models to treat specific interactions of the solvent with organic molecules is another major obstacle, which makes the reliability of computational treatments more and more questionable as the molecule increases in complexity. In our case, on going from colchicides $\mathbf{3}$ and $\mathbf{6}$ to coupling products $\mathbf{2}$ and $\mathbf{5}$, the borderline between what can be treated and what cannot was surpassed. Our analysis of the CD spectra of "monomers" and "dimmers" in various solvents revealed that specific account is needed for each solvent used, a task that we found hard to accomplish. In practice, this borderline in tractability is set by both the characteristics of the CD spectra in solvents of varying properties and by the computational resources. At any event, our study shows that understanding optically active molecules of the complexity of our coupling products needs, from the experimental side, acquiring CD spectra in different solvents, and, from the computational side, establishing and using methodologies able to account for specifically each solvent used.

Our work shows that obstacles faced by the theoretical interpretation of CD spectra get up dramatically. Besides widely documented general difficulties in predicting CD spectra [35], the main asperities at the structural level are identified here in the presence of both hydrophobic and hydrophilic groups in the same molecule, where chirality arises more from the helicity than the asymmetric carbon [12]. This is exacerbated, with respect to the simpler colchicinoids and isocolchicinoids, by the presence of a further node in the helix, at the central bond that connects the two moieties, the importance of which is difficult to predict in condensed phase. All that gives rise to spectral complexities that pose challenges to computational theories and justify establishing for our coupling products a new class of pseudoaromatic compounds.

\section{Materials and Methods}

\section{Materials}

Spectroscopic-grade EtOH, MeOH, MeCN, and DMSO (C. Erba), as well as $\mathrm{Ni}(\mathrm{COD})_{2}$ (Aldrich), were used as such. DMF (C. Erba) was distilled from $\mathrm{CaO}$ and stored over $30 \mu \mathrm{m}$ molecular sieves under Ar. 2,2,2-Trifluoroethanol (Aldrich) was distilled before use. 10-Chlorocolchicide (1) and 9-chloroisocolchicide (4) were prepared according to literature [10].

\section{Techniques}

UV-VIS: Perkin-Elmer Hitachi 200; CD: Jasco J-40AS; IR: Perkin-Elmer Spectrum One FT-IR. ${ }^{1} \mathrm{H}$ NMR: Varian Gemini BB 200, Unity 300, and Inova $600 \mathrm{MHz}$ spectrometers, with TMS as internal reference, with $\mathcal{F}$ values in Hz. Mass spectra: Applied Biosystems Sciex API 4000, MDS Sciex, Concord, Ontario, Canada, triple quadrupole mass spectrometer equipped with a Turbo-V Ionspray source coupled to a Perkin Elmer Series 200 Micro Pump by Flow Injection. Analysis: $200 \mu \mathrm{l} / \mathrm{min}$ under the following experimental conditions: GUR, 10; GS1, 25; GS2, 25; IS Voltage, $5 \mathrm{kv}$, Turbo T, $300^{\circ} \mathrm{C}$; DP, 20 V. HPLC: Jasco Uvidec100-V with SPD-10A Shimadzu UV-VIS detector; column1: Xper-Chrom C18 $5 \mu \mathrm{m} 250 \times 4.6 \mathrm{~mm}$; column 2: Technicol Kromasil C18 250×10 mm, eluent MeCN/water 40:60.

\section{Synthesis of $10,10^{\prime}$-bicolchicide (2)}

Starting from 10-chlorocolchicide (1) (Figure 1A), 10,10'bicolchicide (2) $(57.8 \%$ yield) was obtained as a yellow-orange gummy solid alongside colchicide $(\mathbf{3})$ [11] (26.2\% yield). Spectral data of 2: ${ }^{1} \mathrm{H}$ NMR $\left(300 \mathrm{MHz}, \mathrm{CDCl}_{3}, 25^{\circ} \mathrm{C}, \mathrm{TMS}\right): \delta=7.45(\mathrm{~d}$, $\left.2 \mathrm{H}, \mathrm{H} 12, \mathrm{H} 12^{\prime}, \mathrm{J}=9.6\right), 7.37$ (s, 2H, H8, H8'), 7.23 (d, 2H, H11, $\left.\mathrm{H} 11^{\prime}, \mathcal{J}=9.6\right), 6.96(\mathrm{~d}, 2 \mathrm{H}, \mathrm{NH}, \mathcal{J}(\mathrm{NH}, 7)=6), 6.52(\mathrm{~s}, 2 \mathrm{H}, \mathrm{H} 4$,
$\left.\mathrm{H} 4^{\prime}\right), 4.69$ (ddd, $2 \mathrm{H}, \mathrm{H} 7$ and $\mathrm{H} 7^{\prime}, \mathcal{F}(7, \mathrm{NH})=6, \mathcal{f}=6.6, \mathcal{f}=12$ ), 3.93, 3.89 and 3.70 (three s, $\left.18 \mathrm{H}, \mathrm{OCH}_{3}\right), 2.2-2.5$ (m, 8H, H5, $\left.\mathrm{H} 6, \mathrm{H}^{\prime}, \mathrm{H} 6{ }^{\prime}\right), 2.0\left(\mathrm{~s}, 6 \mathrm{H}, \mathrm{COCH}_{3}\right)$. See Figure $\mathrm{S} 1 .{ }^{13} \mathrm{C}$ NMR $\left(50.3 \mathrm{MHz} \mathrm{CDCl}_{3}, 25^{\circ} \mathrm{C}, \mathrm{TMS}\right): \delta=23.1,29.9,36.5,51.8,56.2$, $61.3,61.7,107.6,125.5,131.5,134.2,135.1,135.6,143.2,150.1$, 151.5, 153.8, 169.5, 184.6. See Figure S2. IR(neat): $v=1660$, $1615,1556,1511,1456,1403,1347,1320,1260$. ESI-MS: m/z $=737.3[\mathrm{M}+\mathrm{H}]^{+}, 759.4[\mathrm{M}+\mathrm{Na}]^{+}$. HRMS: calcd for $\mathrm{C}_{42} \mathrm{H}_{45} \mathrm{~N}_{2} \mathrm{O}_{10}$ 737.3069; found 737.3065.

\section{Synthesis of $9,9^{\prime}$-biisocolchicide (5)}

Starting from 9-chloroisocolchicide (4) (Figure 1B), 9,9'biisocolchicide $(\mathbf{5})(67.1 \%$ yield) was obtained as a yellow-orange gummy solid alongside isocolchicide (6) [11] (10.4\% yield). Spectral data of $\mathbf{5 a}:{ }^{1} \mathrm{H}$ NMR $\left(600 \mathrm{MHz}, \mathrm{CDCl}_{3}, 25^{\circ} \mathrm{C}\right.$, TMS): $\delta=8.54(\mathrm{~d}, 2 \mathrm{H}, \mathrm{NH}, \mathcal{f}(\mathrm{NH}, 7)=7.8), 7.52\left(\mathrm{~s}, 2 \mathrm{H}, \mathrm{H} 8, \mathrm{H} 8^{\prime}\right), 7.35$ $\left(\mathrm{d}, 2 \mathrm{H}, \mathrm{H} 12, \mathrm{H} 12^{\prime}, \mathcal{J}(12,11)=12.6\right), 7.05$ (d, 2H, H11, H11', $\mathcal{f}(11,12)=12.6), 6.56\left(\mathrm{~s}, 2 \mathrm{H}, \mathrm{H} 4, \mathrm{H}^{\prime}\right), 4.44$ (ddd, 2H, H7, H7', $\left.\mathcal{J}(7, \mathrm{NH})=7.8, \mathcal{J}_{7, \operatorname{pro}(\mathrm{R}) 6}=6.6, \mathcal{J}_{7, \operatorname{pro}(\mathrm{S}) 6}=12.6\right), 3.89,3.85$ and 3.72 (three s, $\left.18 \mathrm{H}, \mathrm{OCH}_{3}\right), 2.47$ (dd, 2H, pro(S)H5, pro(S)H5', $\left.\mathcal{J}_{\operatorname{pro}(\mathrm{S}) 5, \operatorname{pro}(\mathrm{R}) 5}=12.5, \quad \mathcal{J}_{\operatorname{pro}(\mathrm{S}) 5, \operatorname{pro}(\mathrm{R}) 6}=6.5\right), 2.38(\mathrm{ddd}, 2 \mathrm{H}$, $\operatorname{pro}(\mathrm{R}) \mathrm{H} 5, \operatorname{pro}(\mathrm{R}) \mathrm{H}^{\prime}{ }^{\prime}, \mathcal{J}_{\operatorname{pro}(\mathrm{R}) 5, \operatorname{pro}(\mathrm{S}) 5}=12.5, \mathcal{J}_{\operatorname{pro}(\mathrm{R}) 5, \operatorname{pro}(\mathrm{R}) 6}=13$, $\left.\mathcal{J}_{\text {pro(R)5,pro(S)6 }}=6.5\right), 2.27$ (dddd, 2H, pro(R)H6, pro(R)H6', $\tilde{\mathcal{F}}_{\operatorname{pro}(\mathrm{R}) 6, \operatorname{pro}(\mathrm{S}) 6}=12.5, \mathcal{J}_{\operatorname{pro}(\mathrm{R}) 6, \operatorname{pro}(\mathrm{S}) 5}=6.5, \mathcal{J}_{\operatorname{pro}(\mathrm{R}) 6, \operatorname{pro}(\mathrm{R}) 5}=13$, $\left.\mathcal{J}_{\text {pro(R)6,7 }}=6.6\right), 2.18\left(\mathrm{ddd}, 2 \mathrm{H}, \operatorname{pro}(\mathrm{S}) \mathrm{H} 6, \operatorname{pro}(\mathrm{S}) \mathrm{H} 6^{\prime}, \mathcal{J}_{\operatorname{pro}(\mathrm{S}) 6, \operatorname{pro}(\mathrm{R}) 6}\right.$ $\left.=12.5, \quad \mathcal{F}_{\text {pro(S)6,pro(R)5 }}=6.5, \quad \mathcal{F}_{\text {pro(S)6,7 }}=12.6\right), 1.94(\mathrm{~s}, 6 \mathrm{H}$, $\left.\mathrm{COCH}_{3}\right)$. Spectral data of $\mathbf{5 b}:{ }^{1} \mathrm{H}$ NMR $\left(600 \mathrm{MHz}, \mathrm{CDCl}_{3}\right.$, $\left.25^{\circ} \mathrm{C}, \mathrm{TMS}\right): \delta=7.62$ (s, $\left.1 \mathrm{H}, \mathrm{H}^{\prime}\right), 7.47$ (s, $\left.1 \mathrm{H}, \mathrm{H} 8\right), 7.40$ (d, $1 \mathrm{H}$, $\mathrm{H} 12, \mathcal{f}(12,11)=12.6), 7.30$ (d, 1H, H12', $\mathcal{J}=12.6), 7.06$ (d, 1H, $\mathrm{H} 1 \mathrm{l}$ or $\left.\mathrm{H}_{1} 1^{\prime}, \mathcal{f}(11,12)=12.6\right), \mathrm{H} 1 \mathrm{l}$ or $\mathrm{H} 11^{\prime}$ overshadowed by $\mathrm{H} 11$ protons of $\mathbf{5 a}, 6.20\left(\mathrm{~s}, 1 \mathrm{H}, \mathrm{H} 4^{\prime}\right), 6.57$ (s, 1H, H4), 5.17 $\left(\mathrm{d}, 1 \mathrm{H}, \mathrm{NH}^{\prime}, \mathcal{f}_{\mathrm{NH}, 7^{\prime}}=7.8\right), 4.92\left(\mathrm{dd}, 1 \mathrm{H}, \mathrm{H} 7^{\prime}, \mathcal{f}_{7, \mathrm{NH}}=7.8\right.$, $\left.\mathcal{f}_{7, \operatorname{pro}(\mathrm{R}) 6}=7.8\right), 4.20\left(\mathrm{ddd}, 1 \mathrm{H}, \mathrm{H} 7, \mathcal{f}_{7, \mathrm{NH}}=7.8, \mathcal{f}_{7, \mathrm{pro}(\mathrm{R}) 6}=6.6\right.$, $\left.\mathcal{f}_{7, \operatorname{pro}(\mathrm{S}) 6}=12.6\right), 3.95,3.93,3.91,3.90,3.76,3.70(\operatorname{six~s}, 18 \mathrm{H}$, $\mathrm{OCH}_{3}$ and $\left.\mathrm{OCH}_{3}{ }^{\prime}\right), 2.74\left(\mathrm{dddd}, 1 \mathrm{H}\right.$, pro(R)H6', $\mathcal{J}_{\operatorname{pro}(\mathrm{R}) 6^{\prime}, \operatorname{pro}(\mathrm{S}) 6^{\prime}}=$ $\left.14.5, \mathcal{F}_{\operatorname{pro}(\mathrm{R}) 6^{\prime}, \operatorname{pro}(\mathrm{S}) 5^{\prime}}=12, \mathcal{J}_{\operatorname{pro}(\mathrm{R}) 6^{\prime}, \operatorname{pro}(\mathrm{R}) 5^{\prime}}=6, \mathcal{J}_{\operatorname{pro}(\mathrm{R}) 6^{\prime}, 7^{\prime}}=7.8\right)$, 2.59 (ddd, $1 \mathrm{H}, \operatorname{pro}(\mathrm{S}) \mathrm{H} 5^{\prime},, 7$ not given), other $\mathrm{H} 5, \mathrm{H} 6, \mathrm{H} 5^{\prime}$ and H6' overshadowed by corresponding protons of $\mathbf{5 a}, 2.17$ (s, 3H, $\left.\mathrm{COCH}_{3}\right), 1.62\left(\mathrm{~s}, 3 \mathrm{H}, \mathrm{COCH}_{3}{ }^{\prime}\right)$. See Figure S3. For $\mathbf{5 b}$, protons labeled by a quotation mark pertain to the molecular moiety with axial configuration $S_{\mathrm{a}}$. These assignments are supported by 2D proton-COSY experiments in $\mathrm{CDCl}_{3} .{ }^{13} \mathrm{C}$ NMR $(50.3 \mathrm{MHz}$, $\left.\mathrm{CDCl}_{3}, 25^{\circ} \mathrm{C}, \mathrm{TMS}\right): \delta=23.3,29.9,38.8,53.0,56.4,61.4,108.1$, $128.7,133.8,135.4,136.4,138.1,140.0,144.5,150.8,154.3$, 170.4, where the two atropisomers cannot be distinguished because of nearly matching signals. See Figure S4. IR(neat): v $=1652,1610,1587,1556,1489,1454,1403,1347,1321$. ESIMS: $\mathrm{m} / \mathrm{z}=737.3[\mathrm{M}+\mathrm{H}]^{+}, 759.4[\mathrm{M}+\mathrm{Na}]^{+}$. HRMS: calcd for $\mathrm{C}_{42} \mathrm{H}_{45} \mathrm{~N}_{2} \mathrm{O}_{10}$ 737.3069; found 737.3069.

\section{Equilibration of atropisomers and CD spectra}

HPLC (column 1 , flow rate $1 \mathrm{~mL} / \mathrm{min}, \lambda 330 \mathrm{~nm}$ ) of a $\mathrm{CHCl}_{3}$ solution of compound $\mathbf{5}$ showed two peaks with retention times $5.65 \mathrm{~min}(\mathbf{5 a})$ and $6.88 \mathrm{~min}(\mathbf{5 b})$ in a relative peak-area ratio 4.9:1. The two atropisomers gave nearly superimposable UV spectra (SPD-10A Shimadzu UV-VIS detector) (Figure 6). The same composition was obtained by collecting eluates at 6.82 and 8.07 min from preparative HPLC (column 2, flow rate $4 \mathrm{~mL} / \mathrm{min}, \lambda$ $330 \mathrm{~nm}$ ), followed by $\mathrm{CHCl}_{3}$ extraction and drying. Following rapid evaporation to dryness under vacuum, and addition of an equal volume of $\mathrm{EtOH}, \mathrm{HPLC}$ analysis showed that the $\mathbf{5 b / 5 a}$ abundance ratio had risen from initial 17:83 to 5:95 in $45 \mathrm{~min}$ at $22^{\circ} \mathrm{C}$. On evaporation of $\mathrm{EtOH}$ to dryness, and addition of an equal volume of $\mathrm{CHCl}_{3}$, the abundance ratio $\mathbf{5 b / 5 a}$ ratio was 
restored to $17: 83$ in $150 \mathrm{~min}$ at $22^{\circ} \mathrm{C}$. A similar behavior was observed in other solvents, as shown in Table 1. Preparative HPLC collection of eluates at the 6.82 and 8.07 min peaks into precooled cuvettes allowed us to record the $\mathrm{CD}$ spectra at $c a 1^{\circ} \mathrm{C}$ for atropisomers $\mathbf{5 a}$ and $\mathbf{5 b}$ in $\mathrm{MeCN} / \mathrm{H}_{2} \mathrm{O}$ 40:60 (Figure 7). The CD spectrum of $\mathbf{5 b}$ was observed to change with time into a spectrum for the equilibrium $\mathbf{5 a / 5 b}$ mixture, the equilibrium being reached overnight. The initial rate of $\mathbf{5 b} \rightarrow \mathbf{5 a}$ transformation could be estimated as $\triangle \mathrm{CD}$ elongation at $350 \mathrm{~nm} / \Delta \mathrm{t}=0.044 \mathrm{~cm} / \mathrm{min}$ at $1^{\circ} \mathrm{C}$.

\section{Theoretical methods used}

Global conformational space search was carried out with program GMMX, based on Steliou's BAKMDL algorithm, which involves a systematic variation of bond lengths, angles, and formal breaking-closure of rings. Force field MMX [18] was used. Repetitive global space search, forth and back, and from different intermediate positions, converged to the same minima, which should therefore be close to the global minimum. Simulated annealing was carried out with software AMBER with GAFF force field [33], driven by a Python script [19]. DFT calculations were carried out with the suite NWChem [30]. TDDFT calculations were carried out with software ORCA [36]. Analysis and plotting of the results was carried out with software SpecDis v1.45 [25].

\section{Supporting Information}

Method S1 Homocoupling of halocolchicides. Found at: doi:10.1371/journal.pone.0010617.s001 (0.03 MB DOC)

Method S2 Cathodic reduction potentials of colchicine, colchicides and bicolchicides.

\section{References}

1. Harada N, Nakanishi K (1983) Circular dichroic spectroscopy. Exciton coupling in organic stereochemistry. University Science Books: California.

2. Berova N, Di Bari L, Pescitelli G (2007) Application of electronic circular dichroism in configurational and conformational analysis of organic compounds. Chem Soc Rev 36: 914-931.

3. DeVoe H (1964) Optical properties of molecular aggregates. I. Classical model of electronic absorption and refraction. J Chem Phys 41: 393-400.

4. DeVoe H (1965) Optical properties of molecular aggregates.II. Classical theory of the refraction, absorption and optical activity of solutions and crystals. J Chem Phys 43: 3199-3208.

5. Frenkel J (1931) On the transformation of light into heat in solids. J Phys Rev 37: $17-44$.

6. Superchi S, Giorgio E, Rosini C (2004) Structural determinations by circular dichroism. Spectra analysis using coupled oscillator methods: an update of the applications of the DeVoe polarizability model. Chirality 16: 422-451.

7. Zandomeneghi M (1979) Circular dichroism of an alkylbenzene. A coupled oscillator approach. J Phys Chem 83: 2926-2928.

8. Gawronski J, Skowronek P (2004) New chromophores for organic stereochemical analysis by exciton-coupled circular dichroism. Current Organic Chemistry 8: $65-82$.

9. Semmelhack MF, Helquist PM, Jones LD (1971) Synthesis with zerovalent nickel. Coupling of aryl halides with bis(1,5-cyclooctadiene) nickel $(0)$. J Am Chem Soc 93: 5908-5910.

10. Cavazza M, Pietra F (1997) A general entry to 10-halocolchicides and 9haloisocolchicides. Synthetic Commun 27: 3405-3413.

11. Danieli B, Lesma G, Palmisano G, Riva R (1985) The structure elucidation of pseudothiocolchicine. Helv Chim Acta 68: 2173-2176.

12. Cavazza M, Zandomeneghi M, Pietra F (2000) Isolation of atropisomers in both the isocolchicide and colchicine series of alkaloids and determination of their chiroptical properties. Tetrahedron Lett 41: 9129-9133.

13. Gaffield W, Lundin RE, Horowitz RM (1984) Conformational isomerism and its relation to the mutarotation of isocolchicine. Chem Commun. pp 610612.

14. Venables DS, Schmuttenmaer CA (1998) Far-infrared spectra and associated dynamics in acetonitrile-water mixtures measured with femtosecond $\mathrm{THz}$ pulse spectrometry. J Chem Phys 108: 4935-4944.

15. Kuyper LF, Hunter RN, Ashton D, Merz KM, Kollman PA (1991) Free energy calculations on the relative solvation free energies of benzene, anisole, and 1,2,3-
Found at: doi:10.1371/journal.pone.0010617.s002 (0.02 MB DOC)

Calculations S1 Quantum mechanical calculations; molecular dynamics.

Found at: doi:10.1371/journal.pone.0010617.s003 (0.09 MB DOC)

Figure S1 ${ }^{1} \mathrm{H}$ NMR spectrum $\left(300 \mathrm{MHz}, \mathrm{CDCl}_{3}\right)$ for compound 2.

Found at: doi:10.1371/journal.pone.0010617.s004 (0.11 MB TIF)

Figure S2 ${ }^{13} \mathrm{C}$ NMR spectrum $\left(75 \mathrm{MHz}, \mathrm{CDCl}_{3}\right)$ for compound 2.

Found at: doi:10.1371/journal.pone.0010617.s005 (0.15 MB TIF)

Figure S3 ${ }^{1} \mathrm{H}$ NMR spectrum $\left(600 \mathrm{MHz}, \mathrm{CDCl}_{3}\right)$ for $5 \mathrm{a}(83 \%)$ and $5 \mathrm{~b}(17 \%)$.

Found at: doi:10.1371/journal.pone.0010617.s006 (0.14 MB TIF)

Figure S4 ${ }^{13} \mathrm{C}$ NMR spectrum $\left(75 \mathrm{MHz}^{\mathrm{CDCl}} \mathrm{C}_{3}\right)$ for $5 \mathrm{a}(83 \%)$ and $5 \mathrm{~b}(17 \%)$.

Found at: doi:10.1371/journal.pone.0010617.s007 (0.15 MB TIF)

\section{Acknowledgments}

Thanks are due to Dr. Torsten Bruhn for generous guidance in the use of software ORCA and SpecDis for CD/UV calculations.

\section{Author Contributions}

Conceived and designed the experiments: TF MC MZ. Performed the experiments: TF MC MZ FP. Analyzed the data: MC MZ FP. Wrote the paper: TF FP. trimethoxybenzene: theoretical and experimental analysis of aromatic methoxy solvation. J Phys Chem 95: 6661-6666.

16. Rapoport H, Lavigne JB (1956) The mutarotation of isocolchicine. J Am Chem Soc 78: 2455-2459.

17. Pietra F (2007) Why colchicine does not show mutarotation. With M05-2X density functional in the realm of tricky natural products. J Phys Org Chem 20: 1102-1107.

18. Gilbert K (2006) A steric energy minimization software. Revision GMMX, Global MMX Serena Software: Bloomington, IN; http://www.serenasoft.com.

19. Bushnell JE (2004-2007) Xanneal, Version 0.7.2; University of California, Santa Barbara, CA; http://bowers.chem.ucsb.edu/computing/index.shtml.

20. Zhao Y, Schultz NE, Truhlar DG (2006) Design of density functionals by combining the method of constraint satisfaction with parametrization for thermochemistry, thermochemical kinetics, and noncovalent interactions. J Chem Theory Comput 2: 364-382.

21. Lessinger L, Margulis TN (1978) The crystal structure of colchicine. A new application of magic integers to multiple-solution direct methods. Acta Cryst B34: 578-584

22. Lessinger L, Margulis TN (1978) The crystal structure of isocolchicine, an inactive isomer of the mitotic spindle inhibitor colchicine. Acta Cryst B34: $1556-1561$.

23. Veracini CA, Pietra F (1972) Molecular structure of tropone from its ${ }^{1} \mathrm{H}$ nuclear magnetic resonance spectrum in a nematic solvent. J Chem Soc Chem Commun. pp 1262-1263.

24. Goerigk L, Grimme S (2009) Calculation of electronic circular dichroism spectra with time-dependent double-hybrid density functional theory. J Phys Chem A 113: $767-776$

25. Bruhn T, Hemberger Y, Schaumlöffel A, Bringmann G (2009) SpecDis version 1.45, University of Wuerzburg,Germany; http://www-organik.chemie.uniwuerzburg.de/lehrstuehlearbeitskreise/bringmann/specdis.

26. Klamt A, Jonas V (1996) Treatment of the outlying charge in continuum solvation models. J Chem Phys 105: 9972-9981.

27. Caricato M, Mennucci B, Tomasi J, Ingrosso F, Cammi R, et al. (2006) Formation and relaxation of excited states in solution: a new time dependent polarizable continuum model based on time dependent density functional theory. J Chem Phys 124: 124520-124533.

28. Cramer CJ, Truhlar DG (2008) A universal approach to solution modeling. Acc Chem Res 41: 760-768. 
29. Florián J, Warshel A (1999) Calculations of hydration entropies of hydrophobic, polar, and ionic solutes in the framework of the Langevin dipoles solvation model. J Phys Chem B 103: 10282-10288.

30. Kendall RA, Apra E, Bernholdt DE, Bylaska EJ, Dupuis M, et al. (2000) High performance computational chemistry: an overview of NWChem a distributed parallel application. Comp Physics Comm. 128: 260-283.

31. Kühne TD, Krack M, Mohamed F, Parrinello M (2007) Efficient and accurate Car-Parrinello-like approach to Born-Oppenheimer molecular dynamics. Phys Rev Lett 98: 066401.

32. Major DT, Gao J (2006) A combined quantum mechanical and molecular mechanical study of the reaction mechanism and $\alpha$-amino acidity in alanine racemase. J Am Chem Soc 128: 16345-16357.
33. Case DA, Darden TA, Cheatham TE, Simmerling CL, Wang J, et al. (2008) AMBER 10; University of California, San Francisco, CA; http://ambermd.org.

34. Elstner M, Porezag D, Jungnickel G, Elsner J, Haugk M, et al. (1998) Selfconsistent-charge density-functional tight-binding method for simulations of complex materials properties. Phys Rev B 58: 7260-7268.

35. Crawford TD, Tam MC, Abrams ML (2007) The current state of $a b$ initio calculations of optical rotation and electronic circular dichroism spectra. J Phys Chem 111: 12057-12068.

36. Petrenko T, Neese F (2007) Analysis and prediction of absorption band shapes, fluorescence band shapes, resonances Raman intensities and excitation profiles using the time-dependent theory of electronic spectroscopy. J Chem Phys 127: 164319 . 